\title{
Low Plasma Total Cholesterol Concentration: A Sensitive Evaluation Marker in Hospitalized Patients with Nutritional Deficiency Malnutrition
}

\author{
Jia Wang, Zhongxin Hong* \\ Department of Clinical Nutrition, Beijing Friendship Hospital, Capital Medical University, Beijing, China
*Corresponding author: hongzhongxin@vip.sina.com
}

Received July 14, 2014; Revised August 23, 2014; Accepted August 28, 2014

\begin{abstract}
Objective: To investigate sensitive evaluation markers in hospitalized patients with nutritional deficiency malnutrition. Methods: A total of 358 hospitalized patients were enrolled. Each patient was analyzed with Subjective Global Assessment (SGA), and was divided into well nutrition group, medium nutrition group and poor nutrition group. The relationships between low plasma total cholesterol (TC) level and other variables were analyzed. Results: Irrespective of men or women, the morbidities of low plasma TC, low serum albumin (ALB), low serum total proteins (TP), low plasma triglyceride (TG) and low hemoglobin (HB) concentration were as same as the result of subjective global assessment (SGA). Plasma TC concentration was positively correlated with serum ALB, HB, plasma TG, lymph cells number (Ly\#), and body mass index (BMI). The level of plasma TC between men and women was different. Logistic regression analysis showed that serum ALB and plasma TC level were significantly independent predictors of SGA. Influence of plasma TC level on SGA was higher than that of serum ALB level. Meanwhile, low plasma TC level was not correlated with age, but it was correlated with total parenteral nutrition (TPN). Conclusions: It can be drew conclusions that low plasma TC concentration is a sensitive evaluation marker in hospitalized patients with nutritional deficiency malnutrition. It is more excellent than traditional variables, such as serum ALB.
\end{abstract}

Keywords: low plasma total cholesterol, nutritional deficiency malnutrition, Subjective Global Assessment(SGA)

Cite This Article: Jia Wang, and Zhongxin Hong, "Low Plasma Total Cholesterol Concentration: A Sensitive Evaluation Marker in Hospitalized Patients with Nutritional Deficiency Malnutrition.” American Journal of Medical Case Reports, vol. 2, no. 9 (2014): 551-555. doi: 10.12691/jfnr-2-9-4.

\section{Introduction}

The phenomenon of nutritional deficiency malnutrition is highly prevalent in hospitalized patients, and it has a strong impact on the prognosis. Although there are many methods to assess the patients' nutritional status, no one or a group of methods is sensitive and specific to response the nutritional status. In recent years, researchers pay more attention on the low plasma total cholesterol (TC) concentration [1,2]. Foreign studies have shown that low plasma TC level is correlated with patients' nutritional status, and it can influence the patients' prognosis exactly $[3,4,5,6]$. The patients' condition can be improved obviously with the level of plasma TC increasing [7]. Therefore, the objective of this study is to explore the predictive value of evaluating the patients' nutritional status by the level of plasma TC and whether the low plasma TC level is a sensitive evaluation marker or not in hospitalized patients with nutritional deficiency malnutrition.

\section{Materials and Methods}

\subsection{Patients}

We selected 358 hospitalized patients from January 1st 2009 to February 1st 2009 in Beijing Friendship Hospital, including 212 men and 146 women. The average age was $(65.7 \pm 17.5) \mathrm{y}$, from 18 to $96 \mathrm{y}$. There were 72 patients with cardiovascular disease, 65 patients with lung disease, 31 patients with cerebral vascular disease, 10 patients with fracture and bone joint disease, 25 patients with gastrointestinal disease, 48 patients with hepatobiliary and pancreatic disease, 23 patients with urinary system disease, 28 patients with blood system disease, 23 patients with diabetes, and 33 patients with malignant tumors. In this study, 312 patients were given oral feeding, 39 patients were given total enteral nutrition (TEN), and 7 patients were given total parenteral nutrition (TPN).

Case inclusion criteria: 1 , age $\geq 18$ y. 2, no serious liver or kidney dysfunction. 3, oral feeding, enteral nutrition or parenteral nutrition single.

Case exclusion criteria: 1 , age $<18$ y. 2 , serious liver or kidney dysfunction. 3 , oral statin drugs. 4, mixed ways of nutrition.

The study was approved by the ethical committee of Beijing Friendship Hospital of Capital Medical University. And the participants had all written the informed consent. 


\subsection{Study Design}

Patients were taken phlebotomize $10 \mathrm{ml}$ in the next day morning after admission. Use automatic biochemical analyzer Olympus 5400 to test each patient's blood, and record the plasma TC, serum albumin (ALB), potassium (K), serum creatinine (SCR) and serum total protein (TP). Patients were taken fingertip blood to test hemoglobin (HB) and lymphocyte count $\left(\mathrm{Ly}^{\#}\right)$ by conventional methods. We used conventional methods to test patients' height and weight. Body mass index (BMI) =weight (kg)/ [height (m) $]^{2}$.

\subsection{Index Definition Criteria}

According to the reference range for each index in our hospital clinical laboratory, low plasma TC level is defined as TC $<3.9 \mathrm{mmol} / \mathrm{L}$, low serum ALB level is defined as ALB $<35 \mathrm{~g} / \mathrm{L}$, low serum TP level is defined as TP $<60 \mathrm{~g} / \mathrm{L}$, low plasma TG level is defined as TG $<$ $0.57 \mathrm{mmol} / \mathrm{L}$, low serum HB level is defined as HB $<120$ $\mathrm{g} / \mathrm{L}$ for man and $\mathrm{HB}<110 \mathrm{~g} / \mathrm{L}$ for woman, low plasma cells level is defined as Ly\# $<0.8 \times 109 / \mathrm{L}$, low serum $\mathrm{K}$ level is defined as $\mathrm{K}<3.5 \mathrm{mmol} / \mathrm{L}$, low SCR level is defined as SCR $<53 \mu \mathrm{mol} / \mathrm{L}$, Low BMI level is defined as BMI $<18.5 \mathrm{~kg} / \mathrm{m}^{2}$.

\subsection{SGA Classification Standards and Quality Control}

Subjective global assessment (SGA) is based on the patient's complete medical history and physical examination. It mainly includes eight aspects: body weight variation in the previous 2 weeks, dietary intake, presence of gastrointestinal symptoms (loss of appetite, nausea, vomiting, and diarrhea), activity condition, stress reaction level, the loss of subcutaneous fat mass, the muscle wasting and ankle edema condition. In this study, SGA standard was determined by different nutrition doctors. Members were familiar with the SGA standard and evaluation method. The nutrition doctors were trained to assess the nutritional status of the patients with the 8- point SGA score according to the protocol. We used a structured evaluation form to record the index and evaluated the nutritional status.

SGA standard: patients who had weight loss and muscle wasting, but had adequate dietary intake recently with the body weight gain, are classified as the well nutrition status. Patients who had moderate weight loss, continued inadequate dietary intake with the body weight continued declining, sexual dysfunction, and moderate stress disorder, are classified as the medium nutrition status. Patients who had severe weight loss, continued inadequate dietary intake, sexual dysfunction and muscle wasting, which are not affected with disease or stress, are classified as the severe nutrition status [8].

\subsection{Statistical Analysis}

In this study, all data were recorded in a database system on a computer, and statistical analysis was performed using SPSS 11.5 package for Windows. Data are expressed as mean \pm SD. The level of significance was set at $\mathrm{P}<0.05$. Each patient was analyzed with SGA, and were divided into three groups: well nutrition group, medium nutrition group and poor nutrition group. The observed variables were divided into normal group and the group lower than normal. The $2 \times 3$ Chi-square test was used to analyze the variables in different genders at different nutritional status. We get the observed variables which were highly consistent with SGA. Ordered categorical outcome logistic regression analysis was used to get the independent variables of SGA, and can compared the sensitivity of the variables. The plasma TC level is a variable on which researchers pay more attention. We used correlation analysis, $t$ test and One-Way ANOVA to study the relationship between observed variables and plasma TC level.

\section{Results}

1. The comparison of nutritional variables in different genders at different nutritional status

Table 1. The variable comparison at different nutritional status in male patients

\begin{tabular}{|c|c|c|c|c|c|c|}
\hline Index & Standard & Well nutrition(n=45) & Medium nutrition(n=108) & Poor nutrition(n=59) & $X^{2}$ & $\mathrm{P}$ \\
\hline \multirow{2}{*}{ ALB } & $<35 g / L$ & 8 & 84 & 39 & \multirow{2}{*}{49.078} & \multirow{2}{*}{$<0.001$} \\
\hline & $\geq 35 \mathrm{~g} / \mathrm{L}$ & 37 & 24 & 20 & & \\
\hline \multirow{2}{*}{ TP } & $<60 \mathrm{~g} / \mathrm{L}$ & 8 & 50 & 19 & \multirow{2}{*}{11.769} & \multirow{2}{*}{0.003} \\
\hline & $\geq 60 \mathrm{~g} / \mathrm{L}$ & 37 & 58 & 40 & & \\
\hline \multirow{2}{*}{ TC } & $<3.9 \mathrm{mmol} / \mathrm{L}$ & 0 & 39 & 53 & \multirow{2}{*}{88.622} & \multirow{2}{*}{$<0.001$} \\
\hline & $\geq 3.9 \mathrm{mmol} / \mathrm{L}$ & 45 & 69 & 6 & & \\
\hline \multirow{2}{*}{ TG } & $<0.57 \mathrm{mmol} / \mathrm{L}$ & 1 & 8 & 11 & \multirow{2}{*}{9.116} & \multirow{2}{*}{0.01} \\
\hline & $\geq 0.57 \mathrm{mmol} / \mathrm{L}$ & 44 & 100 & 48 & & \\
\hline \multirow{2}{*}{$\mathrm{Hb}$} & $<120 \mathrm{~g} / \mathrm{L}$ & 7 & 50 & 31 & \multirow{2}{*}{16.022} & \multirow{2}{*}{$<0.001$} \\
\hline & $\geq 120 \mathrm{~g} / \mathrm{L}$ & 38 & 58 & 28 & & \\
\hline \multirow{2}{*}{ Ly\# } & $<0.8 * 109 / \mathrm{L}$ & 2 & 13 & 10 & \multirow{2}{*}{3.850} & \multirow{2}{*}{0.146} \\
\hline & $\geq 0.8 * 109 / \mathrm{L}$ & 43 & 95 & 49 & & \\
\hline \multirow{2}{*}{ K } & $<3.5 \mathrm{mmol} / \mathrm{L}$ & 1 & 13 & 4 & \multirow{2}{*}{4.246} & \multirow{2}{*}{0.120} \\
\hline & $\geq 3.5 \mathrm{mmol} / \mathrm{L}$ & 44 & 95 & 55 & & \\
\hline \multirow{2}{*}{ Scr } & $<53 \mathrm{umol} / \mathrm{L}$ & 0 & 0 & 0 & \multirow{2}{*}{ - } & \multirow{2}{*}{ - } \\
\hline & $\geq 53 \mathrm{umol} / \mathrm{L}$ & 45 & 108 & 59 & & \\
\hline \multirow{2}{*}{ BMI } & $<18.5 \mathrm{~kg} / \mathrm{m} 2$ & 2 & 9 & 6 & \multirow{2}{*}{1.167} & \multirow{2}{*}{0.559} \\
\hline & $\geq 18.5 \mathrm{~kg} / \mathrm{m} 2$ & 43 & 99 & 53 & & \\
\hline
\end{tabular}


We used $2 \times 3$ Chi-square test to analyze the variables in different genders at different nutritional status. The variable comparisons in group with different nutritional status were shown in Table 1 and Table 2. ALB, TP, TC,
TG and HB in both male and female patients with different nutritional status were significantly different $(\mathrm{P}<0.05)$. In both male and female patients, TC, ALB, TP, TG and HB were highly consistent with SGA.

Table 2. The variable comparison at different nutritional status in female patients

\begin{tabular}{|c|c|c|c|c|c|c|}
\hline Index & Standard & Well nutrition( $n=45)$ & Medium nutrition(n=80) & Poor nutrition( $\mathrm{n}=21)$ & $X^{2}$ & $\mathrm{P}$ \\
\hline \multirow{2}{*}{ ALB } & $<35 g / L$ & 6 & 57 & 15 & \multirow{2}{*}{42.019} & \multirow{2}{*}{$<0.001$} \\
\hline & $\geq 35 \mathrm{~g} / \mathrm{L}$ & 39 & 23 & 6 & & \\
\hline \multirow{2}{*}{$\mathrm{TP}$} & $<60 \mathrm{~g} / \mathrm{L}$ & 4 & 31 & 10 & \multirow{2}{*}{15.290} & \multirow{2}{*}{$<0.001$} \\
\hline & $\geq 60 \mathrm{~g} / \mathrm{L}$ & 41 & 49 & 11 & & \\
\hline \multirow{2}{*}{ TC } & $<3.9 \mathrm{mmol} / \mathrm{L}$ & 1 & 23 & 18 & \multirow{2}{*}{48.708} & \multirow{2}{*}{$<0.001$} \\
\hline & $\geq 3.9 \mathrm{mmol} / \mathrm{L}$ & 44 & 57 & 3 & & \\
\hline \multirow{2}{*}{ TG } & $<0.57 \mathrm{mmol} / \mathrm{L}$ & 1 & 2 & 13 & \multirow{2}{*}{6.451} & \multirow{2}{*}{0.040} \\
\hline & $\geq 0.57 \mathrm{mmol} / \mathrm{L}$ & 44 & 78 & 18 & & \\
\hline \multirow{2}{*}{$\mathrm{Hb}$} & $<110 \mathrm{~g} / \mathrm{L}$ & 3 & 31 & 8 & \multirow{2}{*}{15.508} & \multirow{2}{*}{$<0.001$} \\
\hline & $\geq 110 \mathrm{~g} / \mathrm{L}$ & 42 & 49 & 13 & & \\
\hline \multirow{2}{*}{ Ly\# } & $<0.8 * 109 / \mathrm{L}$ & 1 & 7 & 3 & \multirow{2}{*}{3.366} & \multirow{2}{*}{0.186} \\
\hline & $\geq 0.8 * 109 / \mathrm{L}$ & 44 & 73 & 18 & & \\
\hline \multirow{2}{*}{ K } & $<3.5 \mathrm{mmol} / \mathrm{L}$ & 1 & 12 & 3 & \multirow{2}{*}{5.097} & \multirow{2}{*}{0.078} \\
\hline & $\geq 3.5 \mathrm{mmol} / \mathrm{L}$ & 44 & 68 & 18 & & \\
\hline \multirow{2}{*}{ Scr } & $<53 \mathrm{umol} / \mathrm{L}$ & 0 & 3 & 0 & \multirow{2}{*}{2.527} & \multirow{2}{*}{0.283} \\
\hline & $\geq 53 \mathrm{umol} / \mathrm{L}$ & 45 & 77 & 21 & & \\
\hline \multirow{2}{*}{ BMI } & $<18.5 \mathrm{~kg} / \mathrm{m} 2$ & 5 & 11 & 5 & \multirow{2}{*}{1.933} & \multirow{2}{*}{0.381} \\
\hline & $\geq 18.5 \mathrm{~kg} / \mathrm{m} 2$ & 40 & 69 & 16 & & \\
\hline
\end{tabular}

2. The comparison results between plasma TC level and other variables

We used correlation analysis and test to study the relationship between observed variables and plasma TC level. Table 3, Table 4 showed that TC was correlated with ALB $(r=0.209, P<0.001)$, TG $(r=0.415, P<0.001)$, HB ( $r=0.229, \mathrm{P}<0.001), \mathrm{Ly}^{\#}(\mathrm{r}=0.131, \mathrm{P}=0.012), \mathrm{BMI}$ $(\mathrm{r}=0.170, \quad \mathrm{P}=0.001)$ and gender $(\mathrm{t}=-4.340, \quad \mathrm{P}<0.001)$ significantly. TC was not correlated with age, K, SCR and TG significantly $(\mathrm{P}>0.05)$. The comparison results between TC and other variables were shown in Table 3, Table 4.

Table 3. The comparison results between TC and other variables

\begin{tabular}{ccc}
\hline Table 3. The comparison results between TC and other variables \\
\hline Index & $r$ & $P$ \\
\hline Age & -0.066 & 0.207 \\
ALB & 0.209 & $<0.001$ \\
TP & 0.037 & 0.479 \\
TG & 0.415 & $<0.001$ \\
Hb & 0.229 & $<0.001$ \\
Ly\# & 0.131 & 0.012 \\
K & 0.026 & 0.622 \\
Scr & 0.027 & 0.607 \\
BMI & 0.170 & 0.002 \\
\hline
\end{tabular}

Table 4. The comparison results between plasma TC level and gender

\begin{tabular}{cccc}
\hline & TC & $t$ & $P$ \\
\hline Male & $4.111 \pm 1.152$ & -4.340 & $<0.001$ \\
Female & $4.706 \pm 1.461$ & & \\
\hline
\end{tabular}

3. Plasma TC level in different ways of nutrition

We used One-Way ANOVA to analyze plasma TC level in different nutrition support methods. The statistical data showed that the TC levels in oral feeding group (MS=2.4591, $\mathrm{P}<0.001$ )and TEN group ( $\mathrm{MS}=2.1682$, $\mathrm{P}=0.004)$ were significantly higher than those in TPN group, while plasma TC levels in oral feeding group and TEN group were no significant difference (MS=0.2909,
$\mathrm{P}=0.444)$. Compared with oral feeding and TEN groups, TPN group was prone to cholesterol disorders. The data were shown in Table 5.

Table 5. The correlation between nutrition support methods and plasma TC level

\begin{tabular}{ccccc}
\hline \multirow{2}{*}{ Oral feeding } & & Mean Square & Std. Error & $P$ \\
& TEN & 0.2909 & 0.23887 & 0.444 \\
TEN & TPN & 2.4591 & 0.63585 & $<0.001$ \\
& Oral feeding & -0.2909 & 0.23887 & 0.444 \\
TPN & TPN & 2.1682 & 0.67092 & 0.004 \\
& Oral feeding & -2.4591 & 0.63585 & $<0.001$ \\
& TEN & -2.1682 & 0.67092 & 0.004
\end{tabular}

4. The independent predictors of SGA

We used ordered categorical outcome logistic regression analysis to analyze SGA. The statistical data showed that serum ALB and plasma TC level were significantly independent predictors of SGA. Compared with the traditional variables, such as serum ALB, plasma TC level was more sensitive and excellent. While other observed variables were not introduced in regression equation, they were not independent predictors of SGA.

\section{Discussion}

Malnutrition includes nutritional deficiency malnutrition and nutritional excess malnutrition. There are many clinical evaluation methods to assess the nutritional deficiency malnutrition, such as SGA, ALB, TP, BMI and body fat (\%) in low level. There are also many clinical evaluation methods to assess the nutritional excess malnutrition, such as TC, BMI and body fat (\%) in high level. The phenomenon of nutritional deficiency malnutrition is highly prevalent in hospitalized patients, and it has a strong impact on the prognosis. Although there are many methods to assess nutritional status in 
patients, there is no one or a group of evaluation methods which can assess nutritional status sensitively and specifically. In recent years, researchers pay more attention on the low plasma TC concentration. Foreign studies have shown that low plasma TC level is correlated with patients' nutritional status, and it can influence the patients' prognosis exactly. The main purpose of this study is to explore the predictive value of evaluating the patients' nutritional status by the level of plasma TC and whether the low plasma TC level is a sensitive evaluation marker or not in hospitalized patients with nutritional deficiency malnutrition.

SGA is usually considered to be a variable to screen nutritional deficiency malnutrition [9]. In this study, hospitalized patients were evaluated with SGA. The patients were divided into three groups: well nutrition group, medium nutrition group and poor nutrition group, according to evaluation results and patients' gender. The situation of low plasma TC level, low serum ALB level and BMI lower than normal was statistical analyzed in each group. The analysis result showed that both male and female patients, TC, ALB, TP, TG and HB were highly consistent with SGA $(\mathrm{P}<0.05)$. Correlation analysis showed that plasma TC level was highly correlated with ALB, TG, HB, Ly ${ }^{\#}$ and BMI $(\mathrm{P}<0.05)$, and it had significant difference in different genders, while serum $\mathrm{K}$, SCR, TP and age were no significantly correlated with plasma TC level. Ordered categorical outcome logistic regression analysis showed that ALB and TC were valid variables to assess the nutritional status, and TC was more sensitive than ALB. The study also found that TC was correlated with nutrition support methods. Compared with oral feeding and TEN, the nutrition support method of TPN was prone to cholesterol disorders. As you see, low plasma TC level was a more sensitive variable to assess nutritional deficiency malnutrition in hospitalized patients than traditional variables, such as low serum ALB level.

Why the low plasma TC level is more sensitive to assess nutritional deficiency malnutrition than low serum ALB level? We think there are two possible reasons. Firstly, half-life of cholesterol is 8 days, while that of ALB is 16-20 days. Secondly, serum ALB level is affected by many factors, such as blood volume and renal function, while the influence of plasma TC level is less than ALB.

Most studies have shown that the phenomenon of the low plasma TC occurs in the early stage of the disease [7]. We summarize three possible reasons as follows. Firstly, when the body suffers from disease, particularly serious illness, the body experience many changes, including the decomposition of lipid increase significantly as the body has excessive catabolic and increased energy consumption; the premise material of synthesis of cholesterol scarce, because when the body suffers from serious stress, the usage of sugar is impedimental and the premise material, such as acetyl coenzyme A and ATP, is reduced [10]; the hormones, such as epinephrine, norepinephrine and glucocorticoid, which secrete increase when the body suffers from serious stress, resulting in the body's blood cholesterol clearance capacity enhanced; the cytokines, such as IL-6 and IL-10, which is correlated with the reduction of plasma TC reported by Bonville [11]; and the free radicals, such as oxygen free radicals, which generate increase and act on the bio-film directly or indirectly, resulting in cholesterol on the bio-film depletion. Secondly, the intake of cholesterol is inadequate. The exogenous intake of cholesterol is crucial to maintain the plasma cholesterol level, because the disease itself can cause obstacle of the synthesis of cholesterol. Our previous study has found [12] that hypocholesterolemia group had low-energy, low-protein and the cholesterol intake lower than normal, and they were far below the normal RDA levels. Thirdly, our study showed that gender and TPN had obvious relationship with hypocholesterolemia, but age was not correlated with hypocholesterolemia. The possible reason for this is that our study includes many different diseases and different severity levels of the same disease which may have different degrees of impact on the plasma TC level. We will do further study to perfect our research.

\section{Acknowledgements}

We express our gratitude to all patients who participated in our study. We thank the head nurses who collected the data for our research, and Jianrong Su, the director of laboratory department, who do the biological inspection for this research. We also thank Ye Ye, the doctoral candidate in Emory University in USA, who help us do the statistical analysis. Zhongxin Hong, the corresponding author, designed the experiment and wrote the manuscript. Jia Wang collected cases, designed form in which pantrymen and nurses recorded data, divided the patients into three groups according to SGA assessment results, input the data and used SPSS 11.5 package to analyze the data. Zhihua Liu's responsibility was to contact nurses and collect data. Li Wu and Lihong Zhang divided patients and gave significant advice to the experiment. No conflicts of interest were declared.

\section{References}

[1] Kondrup J, Rasmussen HH. Nutritional risk screening (NRS 2002): a new method based on an analysis of controlled clinical trials [J]. Clin Nutr, 2003, 22(3): 321-36.

[2] Lin Changmei, Lu Qian, Zhou Yujie. Assessment of pre-operative nutrition status of general surgical patients with different methods [J]. Chinese Journal of Clinical Nutrition, 2008, 16 (1): $12-7$.

[3] Monargue-Favard C, Garcia I, Abidi H, et al. Malnourished elderly people and lipid status [J]. J Nutr Health Aging 2002, 6 (6): 370-4.

[4] Bonnefoy M, Abidi H, Jauffret M, et al. Hypocholesterolemia in hospitalized elderly: relations with inflammatory and nutritional stasus [J]. Rev Med Interne 2002, 23 (12): 991-8.

[5] Schupf N, Costa R, Luchsinger J, et al. Relationship between plasma lipids and all-cause mortility in nondemented elderly [J]. J Am Geriatr Soc 2005, 53 (2): 219-26.

[6] Sacks GS, Dearman K, Replogle WH, et al. Use of subjective global assessment to indentify nutrition-associated complications and death in geriatric long-term care facility residents [J]. J Am Coll Nutr 2000, 19 (5): 570-7.

[7] Pavel Vyroubal, Carlo Chiarla, Ivo Giovannini, et al Hypocholesterolemia in critically serious conditions [J]. Biomed Pap Med Fac Univ Palacky Olomouc Czech Repub, 2008,152 (2):181-9.

[8] Barbosa-Silva MC, Barros AJ. Indications and limitations of the use of subjective global assessment in clinical practice: an update [J]. Curr Opin Clin Nutr Metab Care, 2006, 9(3): 263-9.

[9] Putwatana P, Reodecha P, Sirapo-ngam Y, et al. Nutrition screening tools and the prediction of postoperative infectious and wound complications:comparison of methods in presence of risk adjustment [J]. Nutr 2005, 21(6):691-7. 
[10] Bakalar B, Hyspler R, Pachl J, et al. Changes in cholesterol and its precursors during the first days after major trauma [J]. Wien Klin Wochenschr, 2003, 115(21-22): 775-9.

[11] Bonville DA, Parker TS, Levine DM, et al. The relationships of hypocholesterolemia to cytokine concentrations and mortality in chrtically ill patients with systemic inflammatory response syndrome [J]. Surg Infect 2004, 5 (1): 39-49.

[12] Hong Zhongxin, Hua Xin, Wu Li, et al. The investigation and analysis of hypocholesterolemia in hospitalized patients [J]. Chinese Journal of Clinical Nutrition, 2008, 16 (1): 22-5. 